\title{
Front Matter: Volume 11065
}

, "Front Matter: Volume 11065," Proc. SPIE 12065, AOPC 2021: Optical Sensing and Imaging Technology, 1206501 (24 November 2021); doi: $10.1117 / 12.2622804$

SPIE. Event: Applied Optics and Photonics China 2021, 2021, Beijing, China 


\title{
PROCEEDINGS OF SPIE
}

\section{AOPC 2021: Optical Sensing and Imaging Technology}

\author{
Yadong Jiang \\ Qunbo Lv \\ Dong liu \\ Dengwei Zhang \\ Bin Xue \\ Editors
}

20-22 June 2021

Beijing, China

Organized by

University of Electronic Science and Technology of China (China)

Science and Technology on Low-light-level Night Vision Laboratory (China)

Science and Technology on Electro-Optical Information Security Control (China)

Nano-Optoelectronics Laboratory, Department of Electronic Engineering, Tsinghua University (China)

Sponsored by

Chinese Society for Optical Engineering (China)

Published by

SPIE

Volume 12065 
The papers in this volume were part of the technical conference cited on the cover and title page. Papers were selected and subject to review by the editors and conference program committee. Some conference presentations may not be available for publication. Additional papers and presentation recordings may be available online in the SPIE Digital Library at SPIEDigitalLibrary.org.

The papers reflect the work and thoughts of the authors and are published herein as submitted. The publisher is not responsible for the validity of the information or for any outcomes resulting from reliance thereon.

Please use the following format to cite material from these proceedings:

Author(s), "Title of Paper," in AOPC 2021: Optical Sensing and Imaging Technology, edited by Yadong Jiang, Qunbo Lv, Dong Liu, Dengwei Zhang, Bin Xue, Proc. of SPIE 12065, Seven-digit Article CID Number (DD/MM/YYYY); (DOI URL).

ISSN: 0277-786X

ISSN: 1996-756X (electronic)

ISBN: 9781510650053

ISBN: 9781510650060 (electronic)

Published by

SPIE

P.O. Box 10, Bellingham, Washington 98227-0010 USA

Telephone +1 3606763290 (Pacific Time)

SPIE.org

Copyright (C) 2021 Society of Photo-Optical Instrumentation Engineers (SPIE).

Copying of material in this book for internal or personal use, or for the internal or personal use of specific clients, beyond the fair use provisions granted by the U.S. Copyright Law is authorized by SPIE subject to payment of fees. To obtain permission to use and share articles in this volume, visit Copyright Clearance Center at copyright.com. Other copying for republication, resale, advertising or promotion, or any form of systematic or multiple reproduction of any material in this book is prohibited except with permission in writing from the publisher.

Printed in the United States of America by Curran Associates, Inc., under license from SPIE.

Publication of record for individual papers is online in the SPIE Digital Library.

\section{SPIE. DIGITAL}

Paper Numbering: A unique citation identifier (CID) number is assigned to each article in the Proceedings of SPIE at the time of publication. Utilization of CIDs allows articles to be fully citable as soon as they are published online, and connects the same identifier to all online and print versions of the publication. SPIE uses a seven-digit CID article numbering system structured as follows:

- The first five digits correspond to the SPIE volume number.

- The last two digits indicate publication order within the volume using a Base 36 numbering system employing both numerals and letters. These two-number sets start with 00, 01, 02, 03, 04, 05, 06, 07, 08, 09, 0A, OB ... 0Z, followed by 10-1Z, 20-2Z, etc. The CID Number appears on each page of the manuscript. 


\section{Contents}

OPTICAL SENSING AND IMAGING TECHNOLOGY

$1206502 \quad$ FPGA-based real-time cloud detection camera for small satellites [12065-2]

1206503 Research on point cloud wires detection method for helicopter avoidance [12065-3]

$1206504 \quad$ Absorption quantization in the diagnosis of absorption spectroscopy [12065-5]

1206505 Mapping camera boresight monitor system on-orbit accuracy evaluation [12065-6]

$1206506 \quad$ Imaging degradation evaluation on beam-splitting devices [12065-8]

1206507 Automatic checkerboard corner detection combined with gray features and energy minimization (ADGE) [12065-9]

$1206508 \quad$ Evaluation and characterization method of laser spot for all-angle directions [12065-10]

1206509 Resolution-enhanced quantitative phase imaging from transport of intensity equation: mixedtransfer-function [12065-12]

12065 OA Research on intelligent airborne time-domain electromagnetic system based on fixed-wing UAV [12065-14]

12065 OB Development of military unmanned ground vehicles [12065-15]

12065 OC Research on the performance evaluation system of fiber optic current sensor for microcurrent measurement [12065-16]

12065 OD Research on UAV target localization technology based on master INS information transfer alignment [12065-17]

12065 OE Design and development of information processing system for space target detection and recognition [12065-18]

12065 OF Research on sensitive characteristics of tilted fiber grating sensors [12065-19]

12065 OG Intensity image restoration of lidar based on atmospheric scattering model [12065-20]

$12065 \mathrm{OH} \quad$ Magnetic-thermal coupling effect of depolarized interferometric fiber optic gyroscope under low temperature field [12065-21]

12065 Ol Design of AlO $/$ GaAs high-index contrast subwavelength gratings for 940nm VCSELs [12065-26] 
12065 0J Study on target location of non-line-of-sight polarized ultraviolet light [12065-28]

12065 OK Design of a catadioptric near infrared TV camera [12065-29]

12065 OL Homogeneous off-axis reflective infrared imaging system [12065-30]

12065 OM Long focal length and large aperture zoom optical system [12065-31]

12065 ON Safety reinforcement and identification technology of unmanned system flight control command [12065-32]

$1206500 \quad$ Underwater active polarization imaging algorithm based on low-rank sparse decomposition [12065-33]

12065 OP Research on polarization scattering characteristics of metallic materials based on GM-APD [12065-35]

$120650 Q \quad$ Scattering characteristics of targets at different wavelengths based on Kirchhoff scalar approximation theory [12065-38]

12065 OR High temperature imaging dynamic measurement techniques under harsh experimental environment [12065-39]

12065 OS Multi-period automatic fitting of pulse duration improves NMRG longitudinal relaxation time measurement accuracy [12065-41]

12065 OT Development of experimental prototype of the Space Cryogenic Absolute Radiometer [12065-42]

12065 OU Research status and development trend of optical system design for infrared imaging seeker [12065-44]

12065 OV Research on high dynamic flight simulation test technology [12065-45]

12065 OW Analysis on the development status of unmanned combat [12065-46]

12065 OX Robust navigation technology in GNC system [12065-47]

12065 OY Wavelength selection method of unmanned vehicle-mounted multi-wavelength lidar in offroad environment [12065-48]

$120650 Z$ A depth image acquisition platform based on Kinect V2 [12065-49]

1206510 A shadow information recovery technology in 3D visualization of urban remote sensing images [12065-52]

$1206511 \quad$ Research on hardware in the loop simulation test technology of electro-optic system [12065-54] 
1206512 Infrared medium and long wave fusion algorithm based on color mapping of differential information [12065-55]

1206513 Research on infrared imaging simulation technology of ocean scene [12065-56]

1206514 The influence of detector defocus in multi-pixels sensor based computational ghost imaging [12065-57]

1206515 Research on GM-APD lidar intense information correction technology based on target distance [12065-58]

1206516 High saturation and broadband uni-traveling-carrier photodiode for optical communication system [12065-59]

1206517 Minimum resolvable contrast measurement of low signal-to-noise ratio image recognition based on convolutional neural network [12065-60]

1206518 Stereo rectification of Scheimpflug telecentric lenses [12065-61]

1206519 Automated sampling path planning model for large conical workpiece based on laser scanning techniques [12065-62]

$120651 \mathrm{~A}$ 3D vision guided stove picking based on multi-channel image fusion in complex environment [12065-63]

12065 IB ICCD low-light-level image denoising based on robust principal component analysis [12065-65]

12065 1C Improved nonlinear diffusion denoising method based on wavelet transform and contrast sensitivity function for low-light-level images [12065-66]

12065 ID Effective dark-corner noise removal for infrared images based on spatial-temporal features [12065-67]

$12065 \mathrm{IE} \quad$ Hyperspectral image classification with residual learning networks [12065-68]

$12065 \mathrm{IF} \quad$ Adaptive edge detection of noisy images based on the fusion of grayscale and phase consistency [12065-71]

12065 IG Research on x-ray in-situ image processing technology for electric power strain clamp [12065-73]

$12065 \mathrm{lH} \quad$ Large field of view 3D detection with a bionic curved compound-eye camera [12065-74]

$1206511 \quad$ Application and verification of 3D printing technology in the development of space remote sensing camera [12065-75]

$120651 \mathrm{~J} \quad$ Sliding mode line-of-sight stabilization of two-axis photoelectric platform [12065-77]

12065 IK Backend service system of ship detection and plate recognition system based on deep learning [12065-80] 
$120651 \mathrm{~L}$ A method for calculating the thickness of transparent thin films based on transmittance spectrum [12065-82]

12065 1M Research on ultraviolet detector technology based on optical conversion [12065-83]

12065 iN High precision FPGA real-time linear structured light imaging system [12065-84]

1206510 Research on evaluation method of backscattering characteristics of pulsed laser fuse in smoke environment [12065-85]

12065 IP 3D printed lens on optical fiber tip for imaging applications [12065-86]

12065 1Q Road traffic sign recognition based on lightweight neural network [12065-89]

12065 IR The design of visual telescope based on television viewing-aim [12065-90]

12065 is A novel graphene/QDs curved detector for space applications [12065-97]

12065 IT An innovative graphene/QDs photodetector for remote sensing [12065-98]

$120651 \mathrm{U} \quad$ Imaging in turbid water based on 3D Ghost Imaging [12065-100]

$12065 \mathrm{IV}$ Application of digital image correlation algorithm in evaluation of alkali resistant glass for rubidium atomic clock [12065-102]

12065 IW Design and implementation of control and processing software for portable active and passive imaging detection system [12065-103]

$120651 X \quad$ A special operation UAV in urban space [12065-104]

12065 IY Infrared and visible image fusion via NSCT and gradient domain PCNN [12065-107]

1206512 Strategies of precision enhancement for dual-comb time-of-flight distance measurement with nonlinear detection by numerical simulation [12065-109]

1206520 Distributed detection of upheaval buckling for buried pipelines with Brillouin fiber optic sensors [12065-110]

$1206521 \quad$ Fast and high precision alignment of off-axis reflective optical system [12065-11 1$]$

1206522 Coherence retrieval via three-dimensional intensity measurement [12065-112]

1206523 Design and implementation of remote sensing object detection system based on MPSoC [12065-113]

$1206524 \quad$ Variable motion model for lidar motion distortion correction [12065-114] 
1206525 Spatio-temporal super-resolution of sparse aperture array for space situation awareness [12065-117]

$1206526 \quad$ Low-coherence laser-driven fiber optic gyroscope with enhanced scaling factor stability [12065-118]

1206527 Low-light-level image super-resolution reconstruction via deep learning network [12065-119]

1206528 Analysis of groundwater system characteristic in the Badain Jaran desert and its causes based on GF-2 satellite remote sensing data [12065-120]

1206529 Image restoration technology based on GMM [12065-121]

$120652 \mathrm{~A}$ Simulation research on aerodynamic heat radiation of dome in complicated thermal environment [12065-122]

12065 2B Projected area measurement of complex 3D objects based on point cloud data [12065-123]

12065 2C Point cloud smoothing and denoising algorithm based on local domain change factor [12065-131]

12065 2D Design of a beam condense system with simple structure [12065-134]

$120652 \mathrm{E}$ Three-dimensional reconstruction technique based on triangular phase shift and complementary gray code [12065-137]

$120652 F \quad$ A high-precision laser displacement measurement system [12065-139]

$120652 \mathrm{G}$ Improvement of fidelity on quantum blind signature with imprecise quantum measurement [12065-140]

$120652 \mathrm{H} \quad$ The centroid extraction algorithm of sun based on multi-color model [12065-141]

$1206521 \quad$ Optical fiber refractive sensors enhanced by 2D hydrogen doped molybdenum oxide nanodisks [12065-142]

$120652 \mathrm{~J} \quad$ Endoscope blood vessel image enhancement method based on adaptive nonlinear transformation [12065-143]

12065 2K Morphological edge detection algorithm based on hierarchical multi-scale [12065-144]

$120652 \mathrm{~L} \quad$ Laser Schlieren and the applications in flows [12065-147]

$120652 \mathrm{M}$ Three-dimensional shape measurement based on projector defocusing and deep learning [12065-149]

$120652 \mathrm{~N} \quad$ Effects of guard-ring's depth and space on the performance of silicon avalanche photodetector arrays with TCAD simulation [12065-151] 
1206520

$120652 P$

$120652 Q$

$120652 R$

$120652 S$

$120652 T$

$120652 \mathrm{U}$

$120652 V$

$120652 W$

$120652 X$

$120652 Y$

$120652 Z$

1206530

1206531

1206532

1206533

1206534

1206535

1206536

1206537

1206538
Edge detection of reflectance transformation imaging for colorless traces [12065-152]

A method for automatic segmentation of Hela cell imaging in digital holography [12065-153]

A method of auto-determining the center of spectrum based on two-dimensional Hann window function surface fitting in digital holography [12065-154]

High-speed 3D shape measurement from noisy fringe images using deep learning [12065-155]

Infrared and visible light image fusion algorithm based on FCM and guided filter [12065-160]

Portable laser-induced fluorescence spectrum device for metal ions detection [12065-166]

High-sensitive FBG-based adaptive fiber laser acoustic sensing system [12065-167]

PCB chip pin detection based on line structure light [12065-170]

Optimization of resistive anode for a single photon imaging detector [12065-171]

Research on dual-spectrum headband health monitoring system [12065-172]

Research on passive ranging technology based on wave front coding [12065-173]

Absolute phase measurement method with few-patterns [12065-174]

Optical element sub-surface defect detection combining fluorescence and scattering imaging [12065-175]

Fingerprint segmentation of UV induced fluorescence image using fuzzy enhancement [12065-177]

Laser collimation beam expansion design based on the Galileo structure [12065-178]

Feasibility research of fiber optical gyroscope based on entangled photons [12065-180]

Overview and prospect of dam deformation monitoring technology [12065-181]

Peak search algorithm in Brillouin-based strain sensor [12065-184]

Research on free-form surface optimization method based on surface shape transformation [12065-185]

A plug-and-play demosaicing and denoising method on mobile cameras [12065-186]

A study on the terahertz nondestructive testing of foam sandwich structure composites based on morphological filtering [12065-187] 
1206539 Study on environmental worthiness influence of optical fiber coil adhesive in FOG [12065-188]

12065 3A Phosphor film temperature sensor with polymer derived ceramics as high temperature binder [12065-189]

$120653 \mathrm{~B} \quad$ Research on the technology of value transfer infrared radiometer in external field environment [12065-190]

$120653 \mathrm{C} \quad$ Bias instability of additional modulation from interaxial electromagnetic interference in multiaxis fiber optical gyroscope [12065-193]

12065 3D LSTM based video stabilization for object tracking [12065-195]

12065 3E Structure design of intelligent camera for distributed reconfigurable remote sensing satellite system [12065-198]

12065 3F Rotational speed measurement with three photon interference [12065-200]

$120653 G \quad$ Optical window environment adaptation analysis of optical wavefront measurement system [12065-201]

$120653 \mathrm{H} \quad$ All-fiber temperature sensor based on Fabry-Perot interferometer [12065-202]

$1206531 \quad$ A pupil location algorithm based on cascaded Haar feature and OTSU dynamic threshold segmentation [12065-205]

12065 3J Research on dynamic performance evaluation of fiber optic gyroscope based on laser Doppler measurement technology [12065-206]

$120653 \mathrm{~K} \quad$ The temperature error modeling and compensation method of fiber optic gyroscope scaling factor [12065-210]

12065 3L Three-dimensional dynamic non-contact attitude detection system based on optical path deflection technology [12065-211]

$120653 \mathrm{M} \quad$ Imaging quality analysis of navigation and terrain cameras for China's first Mars exploration Tianwen-1 mission [12065-213]

12065 3N Improvement of iris location accuracy in iris recognition [12065-217]

$1206530 \quad$ Finite-key analysis based on neural network of practical wavelength division multiplexed decoy-state quantum key distribution [12065-218]

$120653 \mathrm{P} \quad$ A highlight removal method of components with high reflective surface measurement based on binocular vision [12065-219]

$120653 Q \quad$ Design of ultra-high definition and large aperture lens based on curved surface sensor [12065-220] 
$120653 R \quad$ A method for the design of on-orbit maintainable infrared video processor on GEO orbit [12065-221]

1206535 Intra-cavity absorption gas sensing network based on hybrid DWDM and TDM [12065-222]

$120653 T$ Crop extraction combined with airborne hyperspectral images and deep convolutional neural network [12065-223]

$120653 \mathrm{U}$ Target detection in haze background by applying polarimetric imaging dehazing method [12065-225]

12065 3V Interference effects research on a certain shortwave radio of low frequency wide pulse electric field [12065-227]

12065 3W Power integrity simulation and design optimization of the circuit [12065-228] 